\title{
CdTe PHOTOCONDUCTORS FOR LHC LUMINOSITY MONITORING
}

\author{
Rossa E., Bovet C., Meier D., Schmickler H. \\ CERN, Geneva - CH \\ Verger L., Mongellaz F., Rumen G. \\ LETI (CEA-Technologies Avancées), Grenoble - F
}

\begin{abstract}
Detectors made from CdTe photoconductors are being used with great success in LEP to monitor the vertical beam emittance. They can withstand tremendous irradiation of up to $10 \wedge 13$ Gy from hard X-rays. For the LHC it is proposed to measure the relative luminosity with monitors located inside absorbers $142 \mathrm{~m}$ downstream of the interaction points. There the detectors will receive about $10 \wedge 8$ Gy per year of integrated dose, where the high neutron flux is of particular concern.

Thick-polycristalline-CdTe detectors were recently tested for speed, sensitivity and radiation resistance before and after receiving up to $10 \wedge 15$ neutrons per $\mathrm{cm} \wedge 2$. The test results are presented here, along with a comparison of the calculated charge deposition in Silicon, Diamond and GaAs detectors.
\end{abstract}




\title{
CdTe PHOTOCONDUCTORS FOR LHC LUMINOSITY MONITORING
}

\author{
E. Rossa ${ }^{1}$, C. Bovet, D. Meier, H Schmickler, CERN, Geneva, Switzerland \\ L. Verger, F. Mongellaz, G. Rumen, LETI (CEA-Technologies Avancées), Grenoble, \\ France
}

\begin{abstract}
Detectors made from CdTe photoconductors are being used with great success in LEP to monitor the vertical beam emittance. They can withstand tremendous irradiation of up to $10^{13}$ Gy from hard X-rays. For the LHC it is proposed to measure the relative luminosity with monitors located inside absorbers $142 \mathrm{~m}$ downstream of the interaction points. There the detectors will receive about $10^{8} \mathrm{~Gy}$ per year of integrated dose, where the high neutron flux is of particular concern.
\end{abstract}

Thick-polycristalline-CdTe detectors were recently tested for speed, sensitivity and radiation resistance before and after receiving up to $10^{15}$ neutrons per $\mathrm{cm}^{2}$. The test results are presented here, along with a comparison of the calculated charge deposition in Silicon, Diamond and GaAs detectors.

\section{INTRODUCTION}

The luminosity measurement project for the LHC is described in reference $[1,2,3]$. The main specifications for the detector are the following:

1) The relative luminosity must be measured for each bunch in real time ( $40 \mathrm{MHz}$ acquisition).

2) The device must survive a radiation dose of $10^{8}$ Gy per year for 20 years.

3) The radiation resistance must be such that it can operate for the design luminosity of $10^{34} \mathrm{~cm}^{-2} \mathrm{~s}^{-1}$ with a calculated [2] neutron flux of $4.6 \times 10^{9} \mathrm{~cm}^{-2} \mathrm{~s}^{-1}$.

An ionisation chamber is currently under study [2] to meet the above requirements.

In this paper we describe a new CdTe photoconductive material which also fulfils the above requirements. Detectors using CdTe photoconductors have already been used with great success in LEP to monitor the beam vertical emittance [4]. They can withstand tremendous irradiation by hard X-rays [4] of up to $10^{13} \mathrm{~Gy}$. The range of the used synchrotron radiation was between $2 \mathrm{keV}$ and $1 \mathrm{MeV}$ with the mean value at 200 $\mathrm{KeV}$. The power density reaches 10 watts $/ \mathrm{mm}^{2}$.

\footnotetext{
${ }^{1}$ corresponding author email: edouard.rossa@cern.ch
} 
The photoconductors are made from semiconductor material. The impinging ionising particle (or photon pulse) creates electron-hole pairs along the interaction path. These electron-hole pairs increase the conductivity of the semiconductor, so that an increased current will flow across the path of interaction in the presence of a bias voltage.

A simplified description allows us to say that the current flow turns on with a rise time similar to the duration of the incident ionising pulse and turns off with a rate given by the carrier recombination lifetime. Although most intrinsic semiconductors have carrier lifetimes greater than $100 \mathrm{ps}$, by suitable processing it is possible to reduce these lifetimes to as low as $400 \mathrm{fs}$ [5]-[6].

For very high speed applications, CdTe detectors are usually $[7,8]$ made in thin layers (of a few microns) comprised of small crystals with a decay time of a few picoseconds. LETI [9] has recently developed the deposition of thick-polycristallineCdTe layers (up to $.7 \mathrm{~mm}$ ) with a decay time of a few nanoseconds and with a sensitivity of more than 10'000 collected electrons per MIP (Minimum Ionising Particle).

For the LHC luminosity detector [2] particles have to be detected at a rate of $40 \mathrm{MHz}$, which means that a carrier lifetime of few nanoseconds is sufficient. Thickpolycristalline CdTe detectors have recently been tested for speed, sensitivity and radiation resistance, before and after an irradiation of $10^{15}$ neutrons per $\mathrm{cm}^{2}$.

\section{MATERIAL SELECTION}

The calculated charges created by minimum ionising particles for the most common semiconductors are displayed in the table 1.

- The thickness is fixed at $0.3 \mathrm{~mm}$ for all materials.

- The number of charges created is often much larger than the total number of charges really collected by the input circuit. The number of collected charges depends on the quality of the input circuit and also on the carrier lifetime compared to the carrier transit time between the electrodes in the semiconductor. 


\begin{tabular}{|l|c|c|c|c|}
\hline Thickness .3mm & CdTe & GaAs & Si & Diamond \\
\hline Density $\left[\mathrm{g} / \mathrm{cm}^{3}\right]$ & 5.85 & 5.32 & 2.33 & 3.51 \\
\hline $\begin{array}{l}\text { Mean energy loss } \\
(\mathrm{dE} / \mathrm{dx}) \text { min }\left[\mathrm{MeV} /\left(\mathrm{g} / \mathrm{cm}^{2}\right)\right]\end{array}$ & 1.26 & 1.40 & 1.66 & 1.78 \\
\hline $\begin{array}{l}\text { Mean energy to create } \\
\text { electron-hole pair: }[\mathrm{eV}]\end{array}$ & 4.43 & 4.2 & 3.61 & 13 \\
\hline $\begin{array}{l}\text { Mean number of charges } \\
\text { created in 300 microns }\end{array}$ & 50000 & 53000 & 32200 & 11850 \\
\hline
\end{tabular}

Table 1: Charges created by a Minimum Ionising Particle (MIP) in 0.3-mm thick semiconductors

From Table 1 it can be seen that the number of charges created by 1 MIP in CdTe is large enough to accept a poor collection efficiency. A value of 10000 electrons collected per MIP is usually known as a "comfortable" signal.

The samples made by LETI are discs of CdTe with a diameter of about $17 \mathrm{~mm}$ and with gold electrodes of 7 by $7 \mathrm{~mm}$ on both sides.

Reports on the CVD Diamond Radiation Detector Development are on the web site: http://rd42.web.cern.ch/RD42/.

In the same manner, the latest news on research and development on Silicon for future experiments can be found at the web site: http://rd48.web.cern.ch/RD48/.

\section{RESPONSE SPEED}

The tests of the speed of the CdTe output signal were undertaken [10] at the Laboratoire de Sciences et Ingénierie des surfaces at the Université Claude BernardLyon 1. 
A laser pulse of 35 picoseconds fwhm at $1060 \mathrm{~nm}$ was fired perpendicularly onto the gold plated electrodes. The thin electrodes in these samples were porous enough to allow photons to reach the main bulk of the photoconductor.

The photon transmission through the $.5 \mathrm{~mm}$ thick sample was around $50 \%$ at 1060 $\mathrm{nm}$. Such a pulse was a good simulation of the ionisation track produced by a high energy particle going through the detector parallel to the electric field.

The signal induced by the laser pulse was very large and easy to measure on a single shot, fast-sampling oscilloscope. The rise time was limited by the bandwidth of the scope to a fraction of nanosecond (see Figure 1).

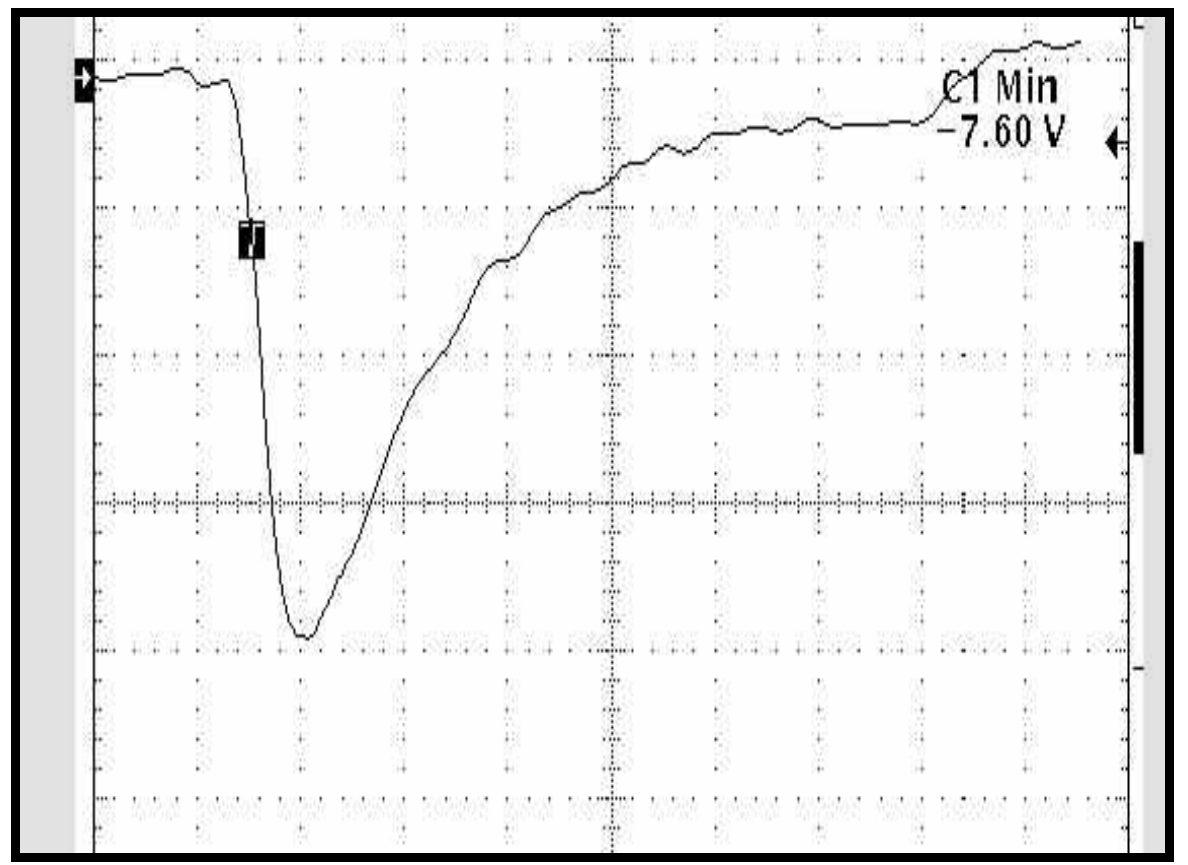

Figure 1: Example of a CdTe output signal just into $50 \Omega$ on the scope. The scale is 2 Volts per division vertically and 2.5 ns per division horizontally.

\section{CHARGE SENSITIVITY MEASUREMENT}

The sensitivity was measured using a charge sensitive amplifier with a shaping time of $2 \mu \mathrm{s}$. The set-up used is described in the reference [11]. The sample was irradiated by a radioactive source ( $\mathrm{Sr} 90$ ). The particles which pass through the sample are detected by a diode and trigger the acquisition of a digital oscilloscope. With a bias voltage of 200 Volts the charge collected reaches 10000 electrons (see Figure 2). 


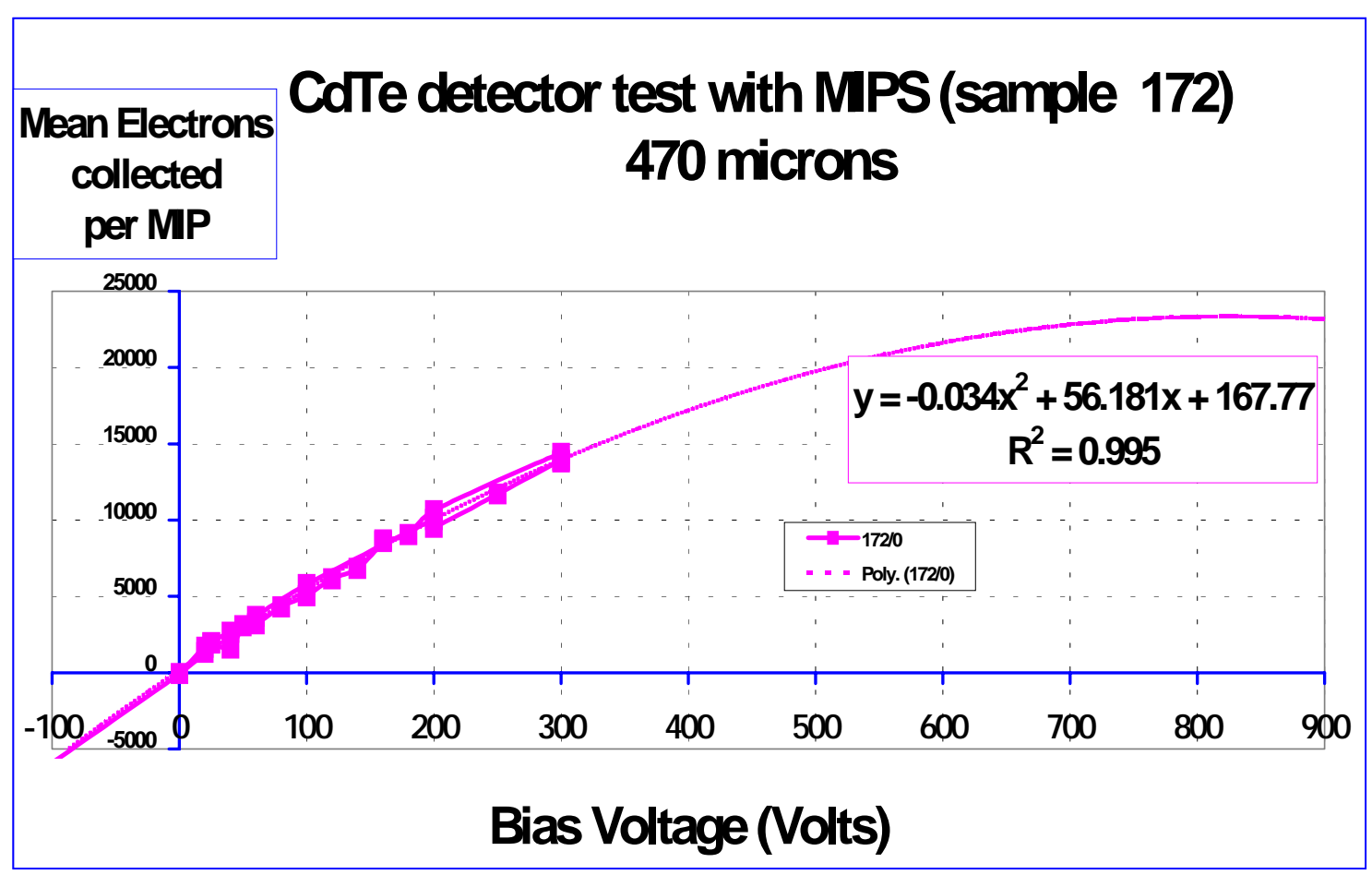

Figure 2: This figure displays the mean number of electrons collected by the charge amplifier connected to the CdTe electrode for each particle detected. The horizontal scale is the bias voltage of the sample under test.

\section{RADIATION RESISTANCE}

The irradiation of the sample was carried out both at CERN and also with the high neutron flux from a reactor in Valduc (France).

The flux of particles and the set-up used at CERN is described in the references [12, 13]. After a total flux of $1.1810^{14}$ neutrons $/ \mathrm{cm}^{2}$ with energy above $1 \mathrm{KeV}$ no significant change in the sample sensitivity was measured. In this beam there was more $8.6610^{13}$ neutrons $/ \mathrm{cm}^{2}$ with energy above $100 \mathrm{KeV}$.

In Valduc the samples were irradiated by up to $10^{15}$ neutrons $/ \mathrm{cm}^{2}$ at $1 \mathrm{MeV}$ energy under the reactor during about 2 weeks. The change in the sensitivity of the CdTe sample was not significant compared to the $5 \%$ precision of the charge collection measurement. Although new calculation [2] give a value of the neutron flux of much larger than the flux at which the detectors have been tested, this first steep is already very encouraging, because no other semiconductor can withstand that flux. 


\subsection{Time response measurement after irradiation by $10^{15}$ neutrons $/ \mathrm{cm}^{2}$}

Figure 3 shows the comparison of the time response measurement before and after irradiation by $10^{15}$ neutrons $/ \mathrm{cm}^{2}$.

The test after neutron irradiation was carried out at CERN with a laser $(1047 \mathrm{~nm}, 60$ ps fwhm) using the same technique outlined in section 3.

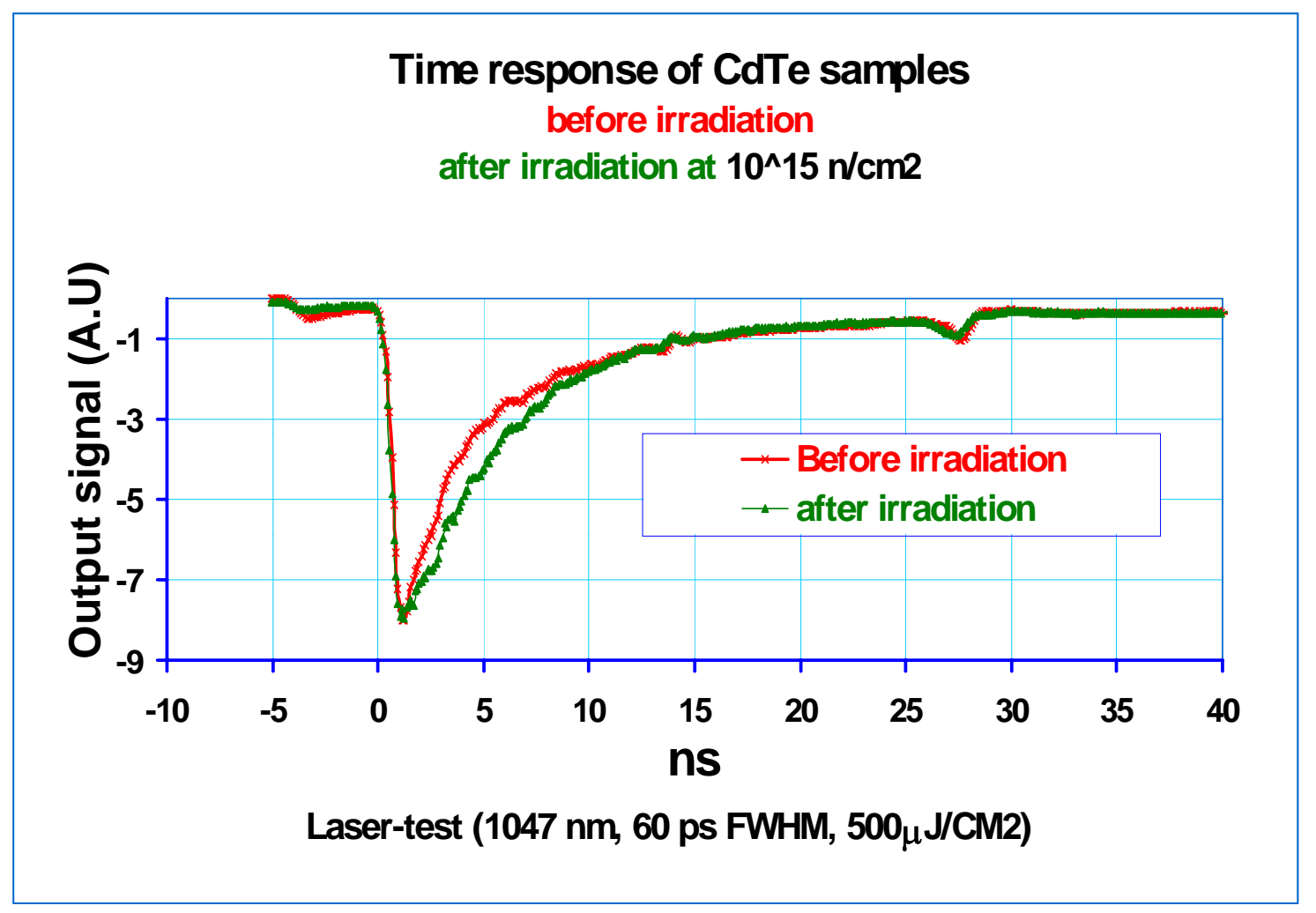

Figure 3: Display of the time response of CdTe samples to a very short laser pulse, before and after irradiation by up to $10^{15}$ neutrons $/ \mathrm{cm}^{2}$.

\subsection{Measurement of the leakage current after irradiation by $10^{15}$ neutrons $/ \mathrm{cm}^{2}$}

Figure 4 shows the leakage current as a function of the bias voltage before and after neutron irradiation. The change in the leakage current due to irradiation is visible at higher bias voltages but the measurement procedure is very critical. As for all high resistance semiconductors the leakage current depends on the bias voltage but has a very large hysteresis. The value measured for the leakage current will therefore depend on the history of the applied voltage. Hence the leakage currents measured at CERN 
were different from the leakage currents measured with a different procedure at the LETI laboratory.

In conclusion, irradiation by $10^{15}$ neutron $/ \mathrm{cm}^{2}$ leads to an increase of the leakage current by a factor of 2 at high bias voltages. There is however always a very good signal to noise ratio.

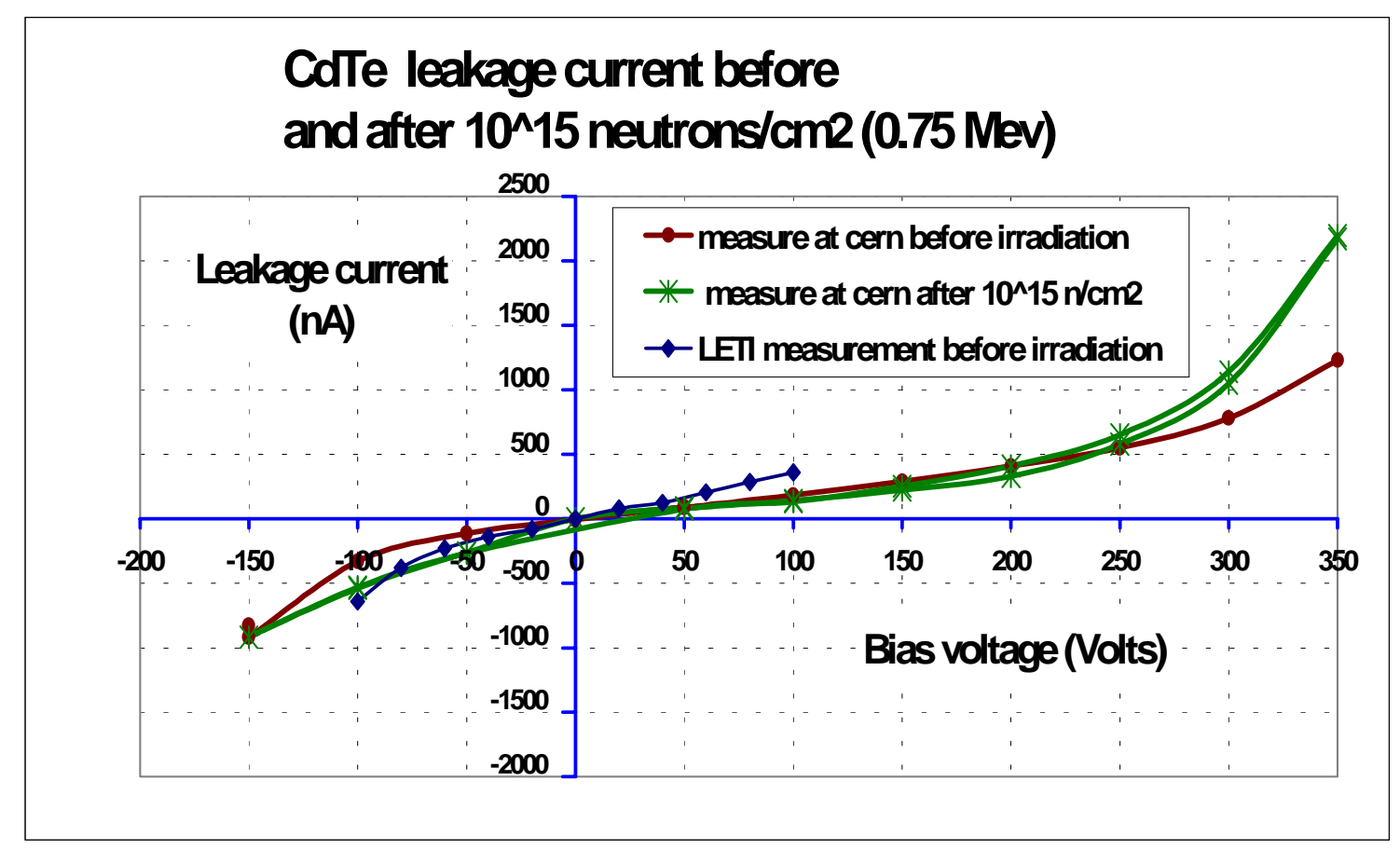

Figure 4: The influence of irradiation on the leakage current, and a comparison of the CERN and LETI measurements.

\subsection{Tests with a fast preamplifier after irradiation by $10^{15}$ neutrons $/ \mathrm{cm}^{2}$}

This measurement was carried out using a digital oscilloscope Lecroy 9354 and a fast preamplifier (DBA) [14] developed in GSI (Germany). The output signal from the detector was sent through a $50 \Omega$ cable to the amplifier using capacitive coupling. The amplified signal was then analysed by the scope. A histogram of the signal amplitude produced by a $\mathrm{Sr} 90$ source in $470 \mu \mathrm{m}$ of polycrystalline CdTe demonstrates a very good signal to noise ratio (see Figure 5). 


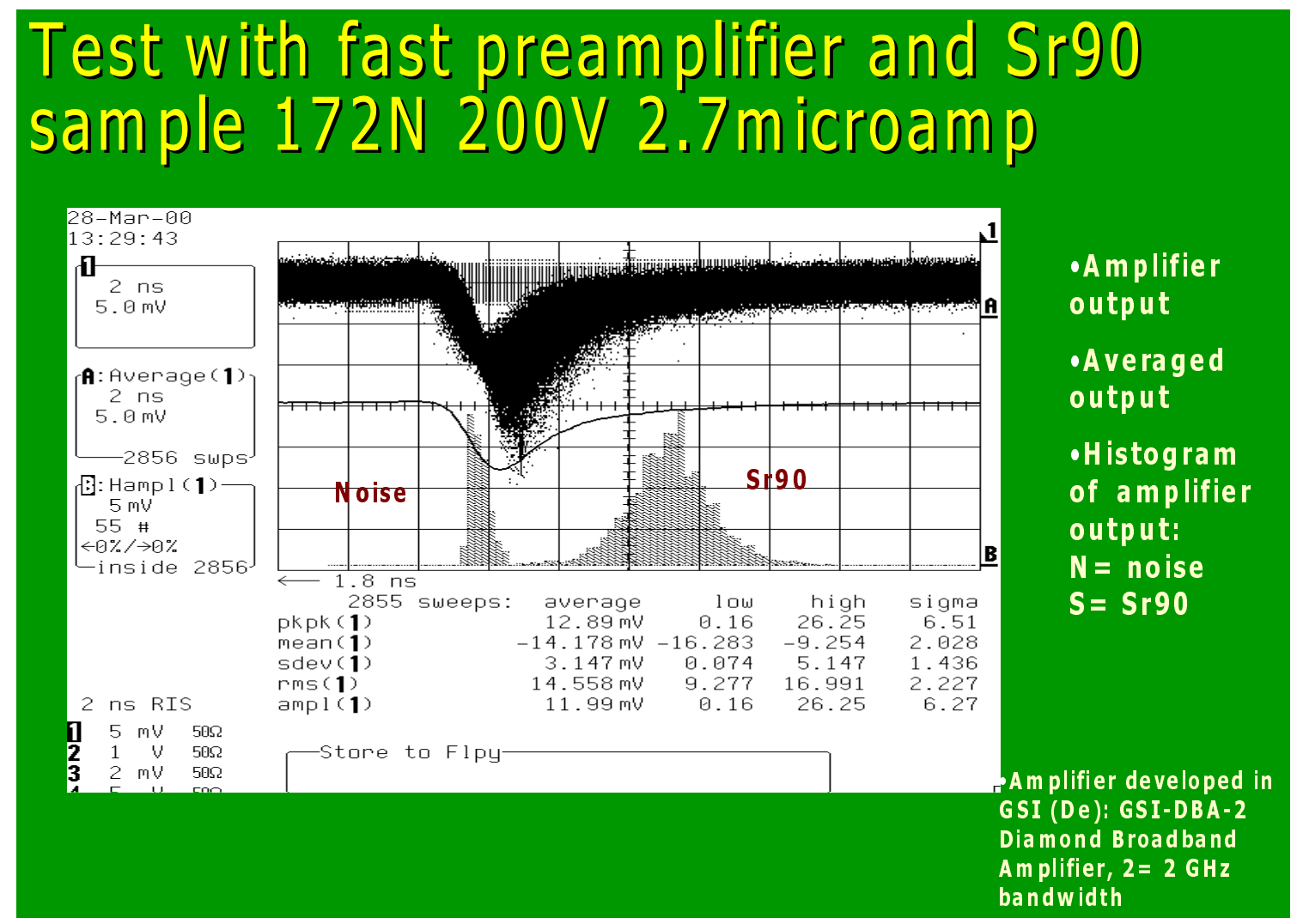

Figure 5: The real time analysis of the preamplified output signal using a Lecroy 9354 oscilloscope.

This preliminary result confirms the speed-tests with infrared laser and seems to show that fast amplifier collects much more charge than the 2 microseconds charge sensitive amplifier.

\section{NEXT STEPS}

Irradiation at higher level under normal bias condition and on-line signal recording will be made in the near future including variations of the operating temperature within the radiation-hard packaging. Also precise calibration of the total charges collected with the fast amplifier compared with charge sensitive electronic must be made using beam test and radioactive sources. 


\section{ACKNOWLEDGEMENT}

The authors would like thank Steve Hutchins and Philippe Legros at CERN and Jean-Pierre Girardeau-Montaut and Belà Kiraly at the University of Lyon for their help during the laser tests. Many thanks also to Eleni Berdermann and Peter Moritz for their very efficient collaboration providing the DBA fast preamplifiers. Last not least we acknowledge the help of Karl Gill during the neutron irradiation tests.

\section{CONCLUSION}

We have successfully tested thick polycrystalline-CdTe detectors for a potential use as LHC luminosity monitors. For this application fast time response (25ns bunch spacing) is required and radiation hardness for neutron irradation: The following results were obtained:

- The signal response of Thick-polycrystalline-CdTe shows a rise-time in the subnanosecond domain and decay time of few ns is hence sufficient for the LHC application.

- A sensitivity of better than 10000 collected electrons per incident minimum ionising particle will generate signal amplitudes which can be easily measured after a simple, fast 50 ohms-preamplifier.

- Irradiation-tests with a neutron dose of about $10^{\wedge} 15 / \mathrm{cm} 2$ have demonstrated no significant loss in sensitivity or speed.

With these outstanding properties thick polycrystalline-CdTe detectors are also potential devices for the LHC detectors or any other particle detectors in a high radiation environment. 


\section{REFERENCES}

[1] W. Turner, E.H. Hoyer and N.V. Mokhov, "Absorbers for High Luminosity Insertions of the LHC", Proc. of EPAC 98, Stockholm, 1998, pg.368.

[2] W.C Turner, M.T. Burks, P.S. Datte, P.F. Manfredi, J.E. Millaud, N.V. Mokhov, M. Placidi, L. Ratti, V. Re, H. Schmickler, V. Speziali, "Development of a detector for bunch by bunch measurement and optimization of luminosity in the LHC", LBNL-45774. CBP Note 337.

[3] See H. Schmickler, "Summary Comments" in Proc. Of Tools for Luminosity Optimisation in LHC, 15-16 Apr. 1999, CERN, Geneva. The proceedings can be found under the web site http://www.cern.ch/CERN/Divisions/SL/groups/bi

[4] R. Jones, A. Manarin, C. Pignard, E. Rossa, H. Schmickler, M. Sillanoli, G Surback, "Real time display of the vertical beam sizes in LEP using the BEXE X-ray detector and fast VME based computers", CERN-SL-99-056-BI, and also: 4th European Workshop on Diagnostics and Instrumentation for Particle Accelerators, Daresbury, UK, 16 - 18 May 1999 / Ed. by Martlew, B G and Smith, R J - Daresbury Lab., Warrington, 2000. - pp.84-87.

[5] C. Martin et al., "Efficient generation of 480 fs electrical pulses on transmission lines by photoconductive switching in metal organic chemical vapor deposited CdTe," Appl. Phys. Lett. 54 (1) 2 January 1989.

[6] X.Q. Zhou et al., "Femtosecond carrier kinetics in low-temperature-grown GaAs", in Proc. 8th Int. Conf. on Ultrafast Phenomena, Antibes-Juan-les-Pins, 1992, Eds. J.L. Martin, A. Migus, G.A. Mourou and A.H. Zewail, Springer Series in Chemical Physics Vol. 55, Springer, Heidelberg (1993).

[7] M. Cuzin, E. Rossa, "Single-shot temporal measurement of short X-ray pulses using very fast photoconductive detectors", in NIM-A 380 (1996), pages 179182.

[8] E. Rossa, "Picosecond diagnostics", in Opt. Eng.: 34 (1995) pp.2353-2360.

[9] LETI (CEA-Technologies Avancées) -17 Rue des Martyrs-F38054 Grenoble Cedex9- France.

[10] With the help of Jean-Pierre Girardeau-Montaut and Bélà Kiraly at the Laboratoire de Sciences et Ingénierie des surfaces at the Université Claude Bernard-Lyon 1. 
[11] Dirk Meier thesis: "CVD Diamond Sensors for Particle Detection and Tracking" Figure 3.27 page 92

http://atlasinfo.cern.ch/Atlas/documentation/thesis/thesis.html

[12] M. Glaser, RADEC99, 5th European Conference on Radiation and its Effects on Components and Systems . by Sharp, Richard ed. ; Publ. Ref.: IEEE Trans. Nucl. Sci. : 47 (2000) no.3, 13 - 17 Sep 1999

[13] M. Glaser, L. Durieu, F. Lemeilleur, M. Tavlet, C. Leroy, P. Roy," New irradiation zones at the CERN-PS " - ROSE Collaboration. In: Nucl. Instrum. Methods Phys. Res., A: 426 (1999) no.1, pp.72-77

[14] The DBA amplifier are developed by Peter Moritz in GSI (Germany) email: p.moritz@gsi.de 